\title{
EXTENSION AND SOME APPLICATIONS OF THE COINCIDENCE THEOREMS
}

\author{
BY \\ ZALMAN RUBINSTEIN( ${ }^{(1)}$ AND J. L. WALSH $\left({ }^{2}\right)$
}

I. Introduction. The purpose of this note is to provide a detailed proof of two coincidence theorems which were introduced in [10], [11], and [12], and proved in particular cases in [10] and [11]. These theorems are then applied to various problems concerning polynomials, exponential polynomials, and certain classes of analytic functions, especially lacunary polynomials. In some cases these results provide analytic proofs to some theorems hitherto treated geometrically.

II. Two lemmas. In this section the function $f(w)=\log (1-w)$ will denote the analytic branch defined in the open unit disk such that $f(0)=0$. Unbounded regions are assumed to contain the point at infinity.

LeMmA 1. Let $r>0 ; m_{k}>0, k=1,2, \ldots, s ; \sum_{k=1}^{s} m_{k}=n$.

(a) If $\alpha_{k}$ are complex numbers, $\left|\alpha_{k}\right| \leqq r$, then there exists a single-calued analytic function $\alpha(z)$ defined for all $|z|>r$ satisfying the relations

$$
\prod_{k=1}^{s}\left(1-\frac{\alpha_{k}}{z}\right)^{m_{k}}=\left(1-\frac{\alpha(z)}{z}\right)^{n}
$$

and $|\alpha(z)| \leqq r$ for $|z|>r$.

(b) If in addition $\sum_{k=1}^{s} m_{k} \alpha_{k}^{l}=0$, for $l=1,2, \ldots, p$, then $|\alpha(z)| \leqq r^{p+1} /|z|^{p}$ for $|z|>r$.

For $p=1$ Lemma 1 was proved in [10, p. 355].

Proof. Let $w=\rho e^{i \phi}, 0<\rho<1$. On the circle $|w|=\rho$ we have

$$
\begin{aligned}
\frac{\partial}{\partial \phi}\left(\frac{\pi}{2}+\phi+\arg f^{\prime}(w)\right) & =\operatorname{Re}\left(1+\frac{w f^{\prime \prime}(w)}{f^{\prime}(w)}\right) \\
& =\frac{1}{|1-w|^{2}}(1-\operatorname{Re} w)>0 .
\end{aligned}
$$

Thus $f(w)$ maps any disk $|w| \leqq \rho$ one to one onto a convex region $R_{\rho}$. For a fixed $z$ $(r<|z|<\infty)$ the function $f(w / z)$ maps the disk $|w| \leqq r$ onto the convex region

Received by the editors August 15, 1968 and, in revised form, June 19, 1969.

${ }^{(1)}$ The research of the first author was partially supported by National Science Foundation.

${ }^{(2)}$ The research of the second author was partially supported by U.S. Air Force, Office of Scientific Research, and Development Command, AFOSR Grant No. 16-1690.

Copyright (C) 1969, American Mathematical Society 
$R_{r /|z|}$. Therefore there exists a unique value $\alpha(z)$ such that

$$
\frac{1}{n} \sum_{k=1}^{s} m_{k} \log \left(1-\frac{\alpha_{k}}{z}\right)=\log \left(1-\frac{\alpha(z)}{z}\right)
$$

and $|\alpha(z)| \leqq r$. Since

$$
\alpha(z)=z\left\{1-\exp \left[\frac{1}{n} \sum_{k=1}^{s} m_{k} \log \left(1-\frac{\alpha_{k}}{z}\right)\right]\right\}
$$

one deduces that $\alpha(z)$ has an analytic continuation to the region $|z|>r$. Thus from equation (1) follows part (a) of the lemma. To establish part (b) we notice that

$$
\begin{aligned}
-\sum_{k=1}^{s} m_{k} \log \left(1-\frac{\alpha_{k}}{z}\right)= & \frac{1}{z} \sum_{k=1}^{s} m_{k} \alpha_{k}+\cdots+p z^{-p} \sum_{k=1}^{s} m_{k} \alpha_{k}^{p} \\
& +\frac{1}{(p+1) z^{p+1}} \sum_{k=1}^{s} m_{k} \alpha_{k}^{p+1}+\cdots \\
= & \frac{1}{z^{p+1}}\left(a_{1}+\frac{a_{2}}{z}+\cdots\right)=-\frac{n \phi(z)}{z^{p+1}}
\end{aligned}
$$

where $\phi(z)$ is analytic in $|z|>r$. Applying equation (1) we have

$$
\alpha(z)=z\left[1-\exp \left(\phi(z) / z^{p+1}\right)\right]=h(z) / z^{p},
$$

where $h(z)$ is analytic in $|z|>r$. For $\varepsilon>0$ let $M_{\varepsilon}=\operatorname{Max}_{|z|=r+\varepsilon}|h(z)|$ and $M=$ $\lim _{\varepsilon \rightarrow 0} M_{\varepsilon}$. We have

$$
M_{\varepsilon} \leqq r(r+\varepsilon)^{p} .
$$

Hence $M \leqq r^{p+1}$. Finally since $M_{\varepsilon} \leqq M$ for all $\varepsilon>0,|h(z)| \leqq M \leqq r^{p+1}$. This concludes the proof of Lemma 1.

The following corollaries follow directly from Lemma 1 .

Corollary 1. If $m_{k}=1, k=1,2, \ldots, s$, then

$$
\prod_{k=1}^{n}\left(z-\alpha_{k}\right)=(z-\alpha(z))^{n}
$$

COROLlaRY 2. If $\beta_{j}(j=1,2, \ldots, n)$ are complex numbers, $\left|\beta_{j}\right| \geqq r$, then there exists a single-valued analytic function $\beta(z)$ defined for all $|z|<r$ satisfying

$$
\prod_{j=1}^{n}\left(z-\beta_{j}\right)=(z \beta(z)-1)^{n} \prod_{j=1}^{n} \beta_{j}
$$

and $|\beta(z)| \leqq 1 / r$ for $|z|<r$.

If in addition $\sum_{k=1}^{n} \beta_{k}^{-l}=0$, for $l=1,2, \ldots, p$, then $|\beta(z)| \leqq|z|^{p} / r^{p+1}$ for $|z|<r$.

Proof. Apply Corollary 1 to $z^{n} Q(1 / z)$, where $Q(z)=\prod_{j=1}^{n}\left(z-\beta_{j}\right)$.

REMARK. It is known [9] that there exists an analogous relation to (2) replacing $\alpha_{k}$ by $\beta_{k}$ and $\alpha(z)$ by $\beta_{1}(z)$, such that $\left|\beta_{1}(z)\right| \geqq r$ for $|z|<r$. However for $p \geqq 1$ this 
estimate cannot be improved to $\left|\beta_{1}(z)\right| \geqq r^{p+1} /|z|^{p}$ as the example $Q(z)=z^{2}-1$, $r=p=\left|\beta_{1}(0)\right|=1$ shows. The function $\left.\alpha(z)=z\left[1-\left(1-z^{-2}\right)^{1 / 2}\right)\right]$ is analytic in $|z|>1$ and satisfies $|\alpha(z)| \leqq|z|^{-1}$. On the other hand neither $\beta(z)=z+i\left(1-z^{2}\right)^{1 / 2}$ nor $b(z)=z-i\left(1-z^{2}\right)^{1 / 2}$ defined and analytic for $|z|<1$ is of modulus at least one for all $|z|<1$. Indeed if for instance $|\beta(z)| \geqq 1$, then $|b(z)| \leqq 1$ but $|b(0)|=1$ and thus we would have a contradiction to the maximum principle. We can therefore conclude that $\beta_{1}(z)$ cannot be analytic in any neighborhood of the origin. Actually one verifies that

$$
\begin{aligned}
\beta_{1}(z) & =\beta(z), \quad \operatorname{Im} z>0 \\
& =b(z), \quad \operatorname{Im} z<0 \\
& =\text { either } \beta(z) \text { or } b(z), \quad \operatorname{Im} z=0 .
\end{aligned}
$$

As we shall see below this difficulty is not encountered if the product of linear factors is replaced by a weighted sum of other reciprocals.

LEMMA 2. With the notation of Lemma 1 we have

(a) If $\left|\alpha_{k}\right| \leqq r$, then there exists an analytic function $\alpha(z)$ defined for all $|z|>r$ satisfying

and $|\alpha(z)| \leqq r$ for $|z|>r$.

$$
\sum_{k=1}^{s} \frac{m_{k}}{z-\alpha_{k}}=\sum_{k=1}^{s} \frac{m_{k}}{z-\alpha(z)}
$$

If in addition $\sum_{k=1}^{s} m_{k} \alpha_{k}^{l}=0$ for $l=1,2, \ldots, p$, then

$$
|\alpha(z)| \leqq r^{p+1} /|z|^{p}, \text { for }|z|>r .
$$

(b) If $\left|\beta_{k}\right| \geqq r$, then there exists an analytic function $\beta(z)$ defined for all $|z|<r$ except possibly for at most $s-1$ poles satisfying

and $|\beta(z)| \geqq r$ for $|z|<r$.

$$
\sum_{k=1}^{s} \frac{m_{k}}{z-\beta_{k}}=\sum_{k=1}^{s} \frac{m_{k}}{z-\beta(z)}
$$

If in addition $\sum_{k=1}^{s} m_{k} \beta_{k}^{-l}=0$ for $l=1,2, \ldots, p$, then

$$
|\beta(z)| \geqq r^{p+1} /|z|^{p} \text { for }|z|<r .
$$

In the case $p=1$, Lemma 2 was proved in [11, p. 355 and p. 365].

Proof. (a) Let $z,|z|>r$, be fixed. The function

$$
g_{z}(w)=\frac{1}{1-w / z}
$$

maps the disk $|w| \leqq r$. onto the interior of a circle which does not include the origin. Thus the argument used in Lemma 1 shows that there exists a unique value $\alpha(z)$ satisfying

$$
\frac{1}{n} \sum_{k=1}^{s} \frac{m_{k}}{1-\alpha_{k} / z}=\frac{1}{1-\alpha(z) / z}
$$


and $|\alpha(z)| \leqq r$. Let $\psi(z)$ denote the left-hand side of (4). Then $\psi(z)$ is analytic in $|z|>r, \psi(\infty)=1$ and $\psi(z) \neq 0$. It follows that

$$
\alpha(z)=z(1-1 / \psi(z))
$$

is analytic in the region $|z|>r$.

To prove the second part of Lemma 2(a) we notice that

$$
\begin{aligned}
\psi(z) & =\frac{1}{n}\left\{n+\frac{1}{z} \sum_{k=1}^{s} m_{k} \alpha_{k}+\cdots+\frac{1}{z^{p}} \sum_{k=1}^{s} m_{k} \alpha_{k}^{p}+\frac{1}{z^{p+1}} \sum_{k=1}^{s} m_{k} \alpha_{k}^{p+1}+\cdots\right\} \\
& =1+\frac{f_{1}(z)}{z^{p+1}}
\end{aligned}
$$

where $f_{1}(z)$ is analytic in $|z|>r$. The function $h_{1}(z)$ defined by

$$
\alpha(z)=\frac{z f_{1}(z)}{z^{p+1}+f_{1}(z)}=\frac{h_{1}(z)}{z^{p}}
$$

is also analytic in $|z|>r$ and one proves that $\left|h_{1}(z)\right| \leqq r^{p+1}$ exactly as in Lemma 1 .

(b) Under the hypotheses made the function $g_{z}(w)$ maps the region $|w| \geqq r$ onto the interior of a circle containing the origin. We can again conclude that there exists a unique value $\beta(z)$ satisfying

$$
\frac{1}{n} \sum_{k=1}^{s} \frac{m_{k}}{1-\beta_{k} / z}=\frac{1}{1-\beta(z) / z}
$$

and $|\beta(z)| \geqq r$ for $|z|<r$. Denote the left-hand side of $(5)$ by $\psi_{1}(z)$. The function $\psi_{2}(z)=z^{-1} \psi_{1}(z)$ is analytic in $|z|<r$. It is also known [4, Theorem $\left.(4,2)\right]$ that the zeros of $\psi_{2}(z)$ are the foci of the curve of class $s-1$ which touches each line segment $\beta_{j} \beta_{k}$ in a point dividing the line segment in the ratio $m_{j} / m_{k}$.

We conclude that the function

$$
\beta(z)=z-\frac{1}{\psi_{2}(z)}
$$

is analytic in $|z|<r$, except possibly for at most $s-1$ poles at the zeros of $\psi_{2}(z)$. To conclude the proof we write

$$
\begin{aligned}
\psi_{2}(z) & =-\frac{1}{n}\left\{\sum_{k=1}^{s} \frac{m_{k}}{\beta_{k}}+z \sum_{k=1}^{s} \frac{m_{k}}{\beta_{k}^{2}}+\cdots+z^{p-1} \sum_{k=1}^{s} \frac{m_{k}}{\beta_{k}^{p}}+z^{p} \sum_{k=1}^{s} \frac{m_{k}}{\beta_{k}^{p+1}}+\cdots\right\} \\
& =z^{p}\left\{b_{1}+b_{2} z+\cdots\right\}=z^{p} h_{2}(z),
\end{aligned}
$$

where $h_{2}(z)$ is analytic in $|z|<r$ and vanishes at most at $s-1$ points. We have

$$
\beta(z)=\frac{1}{z^{p}} \cdot \frac{z^{p+1} h_{2}(z)-1}{h_{2}(z)}=\frac{h_{3}(z)}{z^{p}}
$$

where $h_{3}(z)$ is analytic in $|z|<r$ except possibly for at most $s-1$ poles. Furthermore since $h_{3}(0)=-1 / b_{1} \neq 0$ and $\left|h_{3}(z)\right| \geqq r|z|^{p}$ for $|z|<r$ it follows that $h_{3}(z) \neq 0$ 
in $|z|<r$. Applying the maximum principle to the function $1 / h_{3}(z)$ one deduces easily that $\left|h_{3}(z)\right| \geqq r^{p+1}$ for all $|z|<r$. This concludes the proof of Lemma 2 .

REMARK. It is clear that Lemmas 1 and 2 can also be applied with $p=0$, by regarding the additional conditions imposed on the $\alpha_{k}$ and $\beta_{k}$ as omitted.

III. Some applications of Lemma 1 , to the location of zeros of sections of polynomials. Lemma 1 can be conveniently applied to obtain results on the relative location of zeros of polynomials obtained by various alterations from lacunary polynomials. When $p=0$ some of these results reduce to well-known results and for $p \geqq 1$ they do provide additional information as expected.

THEOREM 1. If all the zeros of $P(z)=a_{0}+a_{q} z^{a}+\cdots+a_{n} z^{n}, 1<q<n$, lie in the region $|z| \geqq r$, then all the zeros of the polynomial $Q(z)=a_{0}+a_{q} z^{q}+\cdots+a_{n-1} z^{n-1}$ lie in the region $|z| \geqq r x(q)$, where $x(q)$ is the positive root of the equation $x^{q}+x-1=0$.

Proof. We apply Corollary 2 to Lemma 1 with $p=q-1$ to $P(z)$. The result follows after a short calculation.

When $q=1, x(1)=\frac{1}{2}$ and the theorem reduces to a result due to Szegö [8].

The example

$$
P(z)=\left(1-z^{q}\right)^{m}=1-m z^{q}+\cdots+z^{q m}
$$

shows that the best value $x_{0}(q)$ of $x(q)$ in Theorem 1 cannot exceed $2^{-1 / q}$. Thus $x(q) \leqq x_{0}(q) \leqq 2^{-1 / q}$. The question of determining $x_{0}(q)$ remains open.

The following result is obtained as above.

THEOREM 2. If all the zeros of the polynomial $P(z)=a_{0} z^{n}+a_{q} z^{n-q}+\cdots+a_{n}$ lie in the disk $|z|<r$ then all the zeros of $P(z)-a_{n}$ lie in the disk $|z|<r / x(q)$.

A repeated application of Theorems 1 and 2 leads to

THEOREM 3. At least one zero of the polynomial

$$
a_{0}+a_{q} z^{q}+a_{v_{1}} z^{v_{1}}+\cdots+a_{v_{k}} z^{v_{k}}
$$

$1 \leqq q<\nu_{1}<\nu_{2}<\cdots<\nu_{k}, a_{q} a_{v_{k}} \neq 0$, lies on the annulus

$$
x^{k}\left(v_{k}-v_{k-1}\right)\left|\frac{a_{v_{k-1}}}{a_{v_{k}}}\right|^{1 /\left(v_{k}-v_{k-1}\right)} \leqq|z| \leqq\left|\frac{a_{0}}{a_{q}}\right|^{1 / q} \cdot x(q)^{-k} .
$$

In this connection compare with a result due to Fejér [4, Corollary $(34,1 b)$, p. 159].

The following two theorems deal with the location of zeros of polynomials obtained from a given polynomial by suppressing alternate terms.

THEOREM 4. If all the zeros of the polynomial

$$
P(z)=a_{0}+a_{q} z^{q}+\cdots+a_{n} z^{n}, \quad a_{0} a_{n} \neq 0, \quad 1 \leqq q \leqq n
$$

lie in the region $|z| \geqq r$, then all the zeros of the polynomial $P(z)+P(-z)$ lie in the region $|z| \geqq r(\sin (\pi / 2 n))^{1 / q}$. 
Proof. If $P\left(z_{0}\right)+P\left(-z_{0}\right)=0$, for some $\left|z_{0}\right|<r$, then by Corollary 2

$$
\left(1-z_{0} \beta\left(z_{0}\right)\right)^{n}+\left(1+z_{0} \beta\left(-z_{0}\right)\right)^{n}=0 \text {. }
$$

For some $\varepsilon_{n}, \varepsilon_{n}^{n}=-1$, we have

Hence

$$
z_{0}=\frac{1-\varepsilon_{n}}{\beta\left(z_{0}\right)+\varepsilon_{n} \beta\left(-z_{0}\right)}
$$

$$
\left|z_{0}\right| \geqq \frac{\left|1-\varepsilon_{n}\right| r^{q}}{2\left|z_{0}\right|^{q-1}} \geqq \sin \frac{\pi}{2 n} \frac{r^{q}}{\left|z_{0}\right|^{q-1}} .
$$

Obviously no estimate of the above type can be obtained for $P(z)-P(-z)$. However, a dual result can be obtained by applying Theorem 4 to $z^{n} Q(1 / z)$. It is interesting to note that if we set $n=k(n) q(n)$, where $k(n)$ is a bounded function of $n$, the zeros of $P(z)+P(-z)$ are in $|z| \geqq r-\varepsilon, \varepsilon>0$, for sufficiently large $n$.

In connection with Theorem 4 we might remark that by using a standard argument involving the Szegö composition theorem we can prove that when $q=1$, when

$$
\begin{aligned}
& Q(z)=P(z)+P(-z), \quad \text { for even } n, \\
& =P(z)-P(-z), \text { for odd } n \text {, }
\end{aligned}
$$

and the zeros of $P(z)$ all lie in the annulus $r \leqq|z| \leqq \rho$, then all the zeros of $Q(z)$ lie in the region

$$
\begin{aligned}
& r \tan (\pi / 2 n) \leqq|z| \leqq \rho \operatorname{ctg}(\pi / 2 n) \text { for even } n \text {, } \\
& 0 \leqq|z| \leqq \rho \operatorname{ctg}(\pi / n) \text { for odd } n \text {. }
\end{aligned}
$$

IV. Some applications of Lemma 2. Lemma 2 is particularly adaptable to application with regard to the location of zeros of linear combinations of lacunary polynomials and their derivatives.

THEOREM 5. If $P(z)=\prod_{k=1}^{n}\left(z-\alpha_{k}\right),\left|\alpha_{k}\right| \leqq r, \sum_{k=1}^{n} \alpha_{k}^{r}=0, l=1,2, \ldots, p$, then all the zeros of the polynomial $c P(z)-P^{\prime}(z)$ lie in the union of the disk $|z| \leqq r$ and the lemniscate of radius $r$ which possesses $p$ foci at the origin and one at the point $n / c$. It is convenient in this connection to consider a lemniscate with a focus at the point at $\infty$ as the empty set.

Proof. If $c P\left(z_{0}\right)-P^{\prime}\left(z_{0}\right)=0$ for some $\left|z_{0}\right|>r$, then by Lemma 2

$$
\frac{n}{z_{0}-\alpha\left(z_{0}\right)}=\sum_{k=1}^{n} \frac{1}{z_{0}-\alpha_{k}}=c \text {. }
$$

Hence

$$
\left|z_{0}^{p}\left(z_{0}-n / c\right)\right|=\left|z_{0}\right|^{p}\left|\alpha\left(z_{0}\right)\right| \leqq r^{p+1} .
$$

For $p=1,(6)$ was proved in $[11$, p. 357]. Similarly one deduces

THEOREM 6. If $P(z)=\prod_{k=1}^{n}\left(z-\beta_{k}\right),\left|\beta_{k}\right| \geqq r, \sum_{k=1}^{n} \beta_{k}^{-l}=0, l=1,2, \ldots, p$, then all the zeros of the polynomial $c P(z)-P^{\prime}(z)$ lie in the exterior of the intersection of the disk and lemniscate defined in Theorem 5. 
V. Estimate of the curvature of lemniscates. In the previous sections we did not make use explicitly of the differential properties of the functions $\alpha(z)$ and $\beta(z)$. In this section we shall apply this property to obtain several estimates on the curvature of level curves of certain analytic functions and particularly the lemniscates.

THEOREM 7. Let $P(w)$ be a function (not necessarily a polynomial) defined in a region containing $1<|w|<\infty$ and such that for $|w|>1$ the function $P^{\prime}(w) / P(w)$ has the representation

$$
\frac{P^{\prime}(w)}{P(w)}=\frac{c}{w-g(w)}
$$

where $g(w)$ is analytic in $|w|>1,|g(w)| \leqq 1$ and $c$ is a real constant.

Let $L_{\mu}=\{w|| P(w) \mid=\mu, \mu>0\}$ and let $K=K(w)$ be the curvature of $L_{\mu}$ at a point $w \in L_{\mu}$, whenever it is defined. Then for $|w| \geqq 3$ the inequality

$$
\left|e^{i \theta} / K-w \operatorname{sign} c\right| \leqq 1
$$

holds, where $\theta$ is the argument of the exterior normal to $L_{\mu}$ at the point $w$.

Proof. If $w=f(z)$ is a univalent function which maps an arc of a circle $|z|=r$ containing the point $z$ onto a curve $\Gamma$ in the $w$-plane, then the curvature of $\Gamma$ at the point $w$ is given by

$$
K=\frac{\left|\operatorname{Re}\left\{1+z\left(f^{\prime \prime}(z) / f^{\prime}(z)\right)\right\}\right|}{\left|z f^{\prime}(z)\right|}
$$

see, e.g. [2]. Letting $f=P^{-1}$ and applying (7) we obtain the following expression for the curvature $K(w)$ of $L_{u}$ at the point $w$ :

$$
\begin{aligned}
K(w) & =\left|\frac{P^{\prime}(w)}{P(w)} \operatorname{Re}\left\{\frac{1-P(w) P^{\prime \prime}(w)}{\left(P^{\prime}(w)\right)^{2}}\right\}\right| \\
& =\left|\frac{\operatorname{Re}\left(1-g^{\prime}(w)\right)}{w-g(w)}\right| .
\end{aligned}
$$

Now

$$
\theta=\arg \frac{P(w)}{P^{\prime}(w)}=\arg \frac{w-g(w)}{c}=\arg (w-g(w))+\arg c .
$$

Inequality (8) can thus be written

$$
\begin{array}{r}
|| \frac{w-g(w)}{\operatorname{Re}\left(1-g^{\prime}(w)\right)}|\exp (i \arg (w-g(w)))-w| \leqq 1, \\
\left|\frac{w-g(w)}{\left|1-\operatorname{Re} g^{\prime}(w)\right|}-w\right| \leqq 1 .
\end{array}
$$

Since $|g(w)| \leqq 1$ in $|w|>1$ we have

$$
\left|g^{\prime}(w)\right| \leqq \frac{1-|g(w)|^{2}}{|w|^{2}-1}
$$


see, e.g. [5, p. 168]. Inequality (10) implies that $\left|g^{\prime}\left(w^{\prime}\right)\right| \leqq 1$ for $|w| \geqq \sqrt{ } 2$. It is therefore sufficient to show that

$$
\frac{\left|w \operatorname{Re} g^{\prime}(w)-g(w)\right|}{1-\operatorname{Re} g^{\prime}(w)} \leqq 1
$$

for $|w| \geqq 3$.

Applying inequality (10) we have

$$
\begin{aligned}
1-\operatorname{Re} g^{\prime}(w) & \geqq 1-\left|g^{\prime}(w)\right| \\
& \geqq 1-\frac{1-|g(w)|^{2}}{|w|^{2}-1} \\
& =\frac{|w|^{2}+|g(w)|^{2}-2}{|w|^{2}-1},
\end{aligned}
$$

and

$$
\begin{aligned}
\left|w \operatorname{Re} g^{\prime}(w)-g\left(w^{\prime}\right)\right| & \leqq\left|w g^{\prime}\left(w^{\prime}\right)\right|+|g(w)| \leqq|w| \frac{1-|g(w)|^{2}}{|w|^{2}-1}+|g(w)| \\
& =\frac{(|w|-|g(w)|)(1+|w||g(w)|)}{|w|^{2}-1} .
\end{aligned}
$$

Thus inequality (8) will hold if

$$
(|w|-|g(w)|)(1+|w||g(w)|) \leqq|w|^{2}+|g(w)|^{2}-2
$$

or equivalently if

$$
|w|^{2}-|w|(1+|g(w)|) \geqq 2+|g(w)| .
$$

Since $|g(w)| \leqq 1$ the last inequality holds obviously for all $|w| \geqq 3$. This completes the proof of Theorem 7 .

Corollary 3. If $w \in L_{u},|w| \geqq 3, c>0(c<0)$, then:

(a) The interior (exterior) normal intersects the unit disk and the radius of curvature lies between the two segments obtained by joining $w$ with the two points (which may coincide) of intersection of the normal with the unit circumference.

(b) In particular the center of curvature lies in the unit disk (or in its reflection in $w$ ).

(c) The curvature $K(w)$ satisfies the inequalities

$$
\frac{1}{|w|+1} \leqq K(w) \leqq \frac{1}{|w|-1}
$$

Using a calculation similar to that performed in the proof of Theorem 7 we can additionally prove:

THEOREM 8. If $w \in L_{u}$, then

$$
K(w) \leqq \frac{1}{|w|-1} \text { if }|w|>1
$$


and

$$
K(w) \geqq \frac{|w|^{2}-2}{\left(|w|^{2}-1\right)(|w|+1)} \text { if }|w| \geqq \sqrt{ } 2 .
$$

Proof. (a) By (9) we have

$$
K(w) \leqq \frac{1+\left|g^{\prime}(w)\right|}{|w|-1} \leqq \frac{1+\frac{1-|g(w)|^{2}}{|w|^{2}-1}}{|w|-1}=\frac{|w|^{2}-|g(w)|^{2}}{\left(|w|^{2}-1\right)(|w|-1)} \leqq \frac{1}{|w|-1}
$$

(b) If $|w| \geqq \sqrt{ } 2$

$$
\begin{aligned}
K(w) & \geqq \frac{1-\left|g^{\prime}(w)\right|}{|w|+1} \geqq \frac{|w|^{2}+|g(w)|^{2}-2}{\left(|w|^{2}-1\right)(|w|+1)} \\
& \geqq \frac{|w|^{2}-2}{\left(|w|^{2}-1\right)(|w|+1)} .
\end{aligned}
$$

COROLlaRY 4. If an arc of $L_{\mu}$ lies in $\{w|| w \mid>\sqrt{ } 2\}$, then this arc does not have any inflection points.

In the particular case when $c>0$ and $L_{\mu}$ are lemniscates Corollary 3 follows also from two theorems due to Shaffer [7, Theorems 3 and 4], where inequality (8) is deduced for a region containing $\{w|| w \mid \geqq 3\}$ but where the number 3 cannot be replaced by a smaller number. However, Corollary 3(c) combined with Theorem 8 contains three out of the four estimates of Theorem 4 in [7]. In addition our Corollary 4 is identical with Corollary 1 in [7], which in turn implies Corollaries $2-4$ of $[7$, p. 66]. We thus conclude that the analytic method which is applicable to a wide class of functions even though it does not yield the strongest possible results in the case of lemniscates is sufficient to yield exact results concerning the sufficient conditions for the convexity of lemniscates. Furthermore, our method provides stronger results when applied to lacunary polynomials, for which we have

Corollary 5. Let $P(w)=\prod_{k=1}^{s}\left(w-\alpha_{k}\right)^{m_{k}}$, where $\left|\alpha_{k}\right| \leqq 1$ and $m_{k}$ are positive integers. If $\sum_{k=1}^{s} m_{k} \alpha_{k}^{l}=0, l=1,2, \ldots, p, p \geqq 1$, then for $|w|>1$

$$
\frac{\left(|w|^{2 p}-p|w|^{p-1}-1\right)|w|^{p}}{\left(|w|^{2 p}-1\right)\left(|w|^{p+1}+1\right)} \leqq K(w) \leqq \frac{|w|^{p+1}+p}{|w|\left(|w|^{p+1}-1\right)}
$$

(b) The lemniscates $L_{\mu}$ are convex if they are contained in the region $|w|>r(p)$, where $r(p)$ is the largest positive root of the equation $x^{2 p}-p x^{p-1}-1=0$.

The right-hand inequality in (a) coincides with the right-hand inequality in Corollary 3(c) for $p=0$ and is sharper than the latter for all $p \geqq 1$. The left-hand inequality in (a) is stronger than the inequality of Theorem $8(b)$ for $p=1$. As regards Corollary $5(\mathrm{~b})$ one can easily show that $r(p) \leqq(p+1)^{1 /(p+1)}$ and $r(2) \leqq \sqrt{ } 2$ so that the estimate for lacunary polynomials is always sharper than for arbitrary polynomials. 
Proof. By Lemma 2 we have $|g(w)| \leqq|w|^{-p}$ for $\left|w^{\prime}\right|>1$. The function $f(w)=g(1 / w)$ is analytic for $|w|<1$ and has the expansion

$$
f(w)=\sum_{k=p}^{\infty} a_{k} w^{k}
$$

Also $|f(w)|<1$ for $|w|<1$. Therefore by a theorem due to Goluzin [1, p. 290]

or equivalently

$$
\left|f^{\prime}(w)\right| \leqq \frac{p|w|^{p-1}}{1-|w|^{2 p}}\left(1-|f(w)|^{2}\right) \text { for }|w|<1
$$

$$
\left|g^{\prime}(w)\right| \leqq \frac{p|w|^{p-1}}{|w|^{2 p}-1}\left(1-|g(w)|^{2}\right) \quad \text { for }|w|>1
$$

Now by (9), Lemma 2 and the last inequality

$$
K\left(w^{\prime}\right) \geqq \frac{1-\left|g^{\prime}(w)\right|}{|w|+|g(w)|} \geqq \frac{|w|^{p}\left(|w|^{2 p}-p|w|^{p-1}-1\right)}{\left(|w|^{p+1}+1\right)\left(|w|^{2 p}-1\right)} .
$$

In particular $K(w)>0$ for $|w|>r(p)$. To complete the proof we notice that by (9)

Applying the inequality

$$
K(w) \leqq\left|\frac{P^{\prime}(w)}{P(w)}-\frac{P^{\prime \prime}(w)}{P^{\prime}(w)}\right| .
$$

$$
\left|\frac{P^{\prime}(w)}{P(w)}-\frac{P^{\prime \prime}(w)}{P^{\prime}(w)}-\frac{1}{w}\right| \leqq \frac{p+1}{|w|\left(|w|^{p+1}-1\right)},
$$

from [6, Theorem 3], we obtain

$$
K(w) \leqq \frac{p+1}{|w|\left(|w|^{p+1}-1\right)}+\frac{1}{|w|}=\frac{|w|^{p+1}+p}{|w|\left(|w|^{p+1}-1\right)} .
$$

This completes the proof.

VI. Sufficient conditions for the stability of exponential polynomials. Recently, Krall [3], has pointed out that there are no suitable techniques to determine whether or not an exponential polynomial of the form

$$
F(z)=z^{n}+a z^{n-1}+\cdots-K e^{i \theta} e^{-\imath z}\left(z^{m}+b z^{m-1}+\cdots\right),
$$

where $0<\tau, 0<K$, and $0 \leqq \theta$ (a,b, etc. are complex constants), is stable, i.e., all its zeros have negative real parts. He proves, among other things, that only for $m<n$, or for $m=n$ and $K<1$ has the function $F(z)$ a finite number of zeros with positive real parts.

The purpose of this section is to give a sharp estimate for the real part of the zeros of $F(z)$ for $m \leqq n$, and to indicate a criterion of stability if in addition $K<1$.

THEOREM 9. Let $F(z)$ be as in (11), $m \leqq n$. Suppose that the polynomial $P(z)=$ $z^{n}+a z^{n-1}+\cdots$ has all its zeros in the disk $|z| \leqq r_{1}$, and that the polynomial $Q(z)=$ 
$z^{m}+b z^{m-1}+\cdots$ has all its zeros in the disk $|z| \leqq r_{2}$. Let $R, R>r_{1}$, satisfy the inequality:

$$
\log \left[\left(R-r_{1}\right)^{n} /\left(R+r_{2}\right)^{m}\right]+\tau R \geqq \log K ;
$$

then all the zeros of $F(z)$ lie in the half-plane $\operatorname{Re} z \leqq R$.

Proof. By Corollary 1, equation (11) can be written in the form:

$$
F(z)=(z-\alpha)^{n}-K e^{i \theta} e^{-\tau z}(z-\beta)^{m},
$$

where $|\alpha(z)| \leqq r_{1}$, and $|\beta(z)| \leqq r_{2}$. If $F\left(z_{0}\right)=0$, with $\operatorname{Re} z_{0}>R$, then

$$
\begin{aligned}
K e^{-\imath R} & >K e^{-\tau \operatorname{Re} z_{0}}=\left|z_{0}-\alpha\right|^{n} /\left|z_{0}-\beta\right|^{m} \geqq\left(\left|z_{0}\right|-r_{1}\right)^{n} /\left(\left|z_{0}\right|+r_{2}\right)^{m} \\
& \geqq\left(R-r_{1}\right)^{n} /\left(R+r_{2}\right)^{m} .
\end{aligned}
$$

Since, for $m \leqq n,\left(x-r_{1}\right)^{n} /\left(x+r_{2}\right)^{m}$ is an increasing function of $x$ for $r_{1}<x$, we get a contradiction to (12) which proves the theorem.

The estimate of the theorem is sharp, as the example:

$$
\theta=0, \quad P(z)=\left(z-z_{1}\right)^{n}, \quad Q(z)=\left(z-z_{2}\right)^{m}, \quad\left(r_{1}-r_{2}\right)^{m}>0,
$$

shows, since in this case $F(z)$ has a real zero greater than $r_{1}$, for which both sides of equation (12) are equal.

Corollary 6. If $F(z), P(z)$, and $Q(z)$ are as in Theorem 9, but if the zeros of $P(z)$ and $Q(z)$ lie in the disks $|z-c| \leqq r_{1}$ and $|z-c| \leqq r_{2}$ respectively, and if $\operatorname{Re} c$ $\leqq-R<-r_{1}$, where $R$ satisfies the inequality:

$$
\log \left[\left(R-r_{1}\right)^{n} /\left(R+r_{2}\right)^{m}\right]+\tau(R+\operatorname{Re} c) \geqq \log K,
$$

then the exponential polynomial $F(z)$ is stable.

This is shown by applying Theorem 9 to the function $F(z+c)$. It follows that the zeros of $F(z+c)$ satisfy $\operatorname{Re} z \leqq R$, hence $\operatorname{Re}(z+c) \leqq R+\operatorname{Re} c \leqq 0$.

For $n=m$, inequality (15) could be satisfied only if $K<1$, which is in accordance with the general theorems in [3].

As in the previous sections somewhat sharper results can be obtained if the polynomials defining $F(z)$ are lacunary.

VII. Domains of starlikeness and convexity for lacunary polynomials. This section deals with a sharp estimate of the largest disks about the origin in which all lacunary polynomials $P(z)$ of degree $n$ normalized by $P(0)=0, P^{\prime}(0)=1$ are starlike or convex.

THEOREM 10. Let $P(z)=z+a_{p} z^{p}+\cdots+a_{n} z^{n}, a_{n} \neq 0, n \geqq p \geqq 2$, and let $z_{1}, z_{2}, \ldots$, $z_{n-1}$ and $z_{1}^{\prime}, z_{2}^{\prime}, \ldots, z_{n-1}^{\prime}$ denote the nonvanishing zeros of $P(z)$ and $P^{\prime}(z)$ respectively. Then

(a) $P(z)$ is convex in the disk $|z| \leqq r n^{-1 /(p-1)}$, where $r=\operatorname{Min}_{1 \leqq k \leqq n-1}\left|z_{k}^{\prime}\right|$. 
(b) $P(z)$ is starlike with respect to the origin in the disk $|z| \leqq \rho n^{-1 /(p-1)}$, where $\rho=\operatorname{Min}_{1 \leqq k \leqq n-1}\left|z_{k}\right|$.

(c) The limits in (a) and (b) are attained by the polynomial $P(z)=z-z^{n} / n, p=n$.

Proof. We apply Lemma 2 (b) with $p$ replaced by $p-2$ to the polynomials $P(z)$ and $P^{\prime}(z)$. We then have

$$
\begin{aligned}
\operatorname{Re}\left(1+z \frac{P^{\prime \prime}(z)}{P^{\prime}(z)}\right) & =\operatorname{Re}\left(1+\sum_{k=1}^{n-1} \frac{z}{z-z_{k}^{\prime}}\right) \\
& =1+\operatorname{Re}\left[\frac{(n-1) z}{z-\alpha(z)}\right],
\end{aligned}
$$

where $|z|<r$ and $|\alpha(z)| \geqq r^{p-1} /|z|^{p-2}$. It follows that

$$
\begin{aligned}
\operatorname{Re}\left(1+z \frac{P^{\prime \prime}(z)}{P^{\prime}(z)}\right) & \geqq 1-\frac{(n-1)|z|}{r^{p-1} /|z|^{p-2}-|z|} \\
& =1-\frac{(n-1)|z|^{p-1}}{r^{p-1}-|z|^{p-1}} .
\end{aligned}
$$

Thus

$$
\operatorname{Re}\left(1+z P^{\prime \prime}(z) / P^{\prime}(z)\right) \geqq 0
$$

if $r^{p-1}-n|z|^{p-1} \geqq 0$. Similarly

$$
\operatorname{Re}\left(z \frac{P^{\prime}(z)}{P(z)}\right)=\operatorname{Re}\left(1+\sum_{k=1}^{n-1} \frac{z}{z-z_{k}}\right)
$$

and the calculation is the same as above replacing $z_{k}^{\prime}$ by $z_{k}$. For the extremal polynomial we have

$$
\begin{aligned}
1+\operatorname{Re}\left(z \frac{P^{\prime \prime}(z)}{P^{\prime}(z)}\right) & =\operatorname{Re} \frac{1-n z^{n-1}}{1-z^{n-1}} \\
\operatorname{Re}\left(z \frac{P^{\prime}(z)}{P(z)}\right) & =\operatorname{Re} \frac{1-z^{n-1}}{1-n^{-1} z^{n-1}}
\end{aligned}
$$

and by substituting $\zeta=z^{n-1}$, one shows easily that the above mentioned disks are the largest possible disks for which $P(z)$ is convex and starlike respectively.

COROLlARY 7. Let $P(z)=z+a_{p} z^{p}+\cdots+a_{n} z^{n}, a_{n} \neq 0,2 \leqq p \leqq n$, and let $r$ and $\rho$ be defined as in Theorem 10. Then $P(z)$ is univalent in the disk

$$
|z| \leqq n^{-1 /(p-1)} \operatorname{Max}(r, \rho) .
$$

In particular $r \geqq \rho n^{-1 /(p-1)}, \rho \geqq r n^{-1 /(p-1)}$, that is

$$
n^{-1 /(p-1)} \leqq r / \rho \leqq n^{1 /(p-1)} .
$$

The right-hand inequality is sharp for all $n$, and the upper bound is attained for $p_{n}(z) \equiv z-z^{n} / n$. The left-hand inequality is not sharp for the case $p=2$. 
VIII. Application of $\S$ II with arbitrary center of gravity of the $\alpha_{k}$. Lemma 1 and Lemma 2 have shown themselves quite useful [9]-[12] in studying zeros of polynomials; they are now to be generalized and applied by making further use of a possible arbitrary center of gravity of the points $\alpha_{k}$. From Lemma 2(a) there follows without difficulty [11]

THEOREM 11. Suppose we have $\left|\alpha_{k}-\alpha_{0}\right| \leqq r, m_{k}>0, \sum_{k=1}^{n} m_{k}=1,\left|z-\alpha_{0}\right|>r$. If $z$ is a zero of the equation

then we have also

$$
\sum_{k=1}^{n} \frac{m_{k}}{z-\alpha_{k}}=A, \quad A \neq 0,
$$

$$
\left|z-\left(\alpha_{0}+1 / A\right)\right| \leqq r .
$$

Use of Lemma 2(b) instead of Lemma 2(a) yields [12]

THEOREM 12. If in Theorem 11 we have $\sum_{k=1}^{n} m_{k} \alpha_{k}=\alpha_{0}$, then we may replace (16) by

$$
\left|\left(z-\alpha_{0}\right)\left[z-\left(\alpha_{0}+1 / A\right)\right]\right| \leqq r^{2} .
$$

We no longer require henceforth that the center of gravity of the weighted points $\alpha_{k}$ be the center of the disk $|z| \leqq 1$ containing the points $\alpha_{k}$ and shall prove

THEOREM 13. Suppose we have $\left|\alpha_{k}\right| \leqq 1, m_{k}>0, \sum_{k=1}^{n} m_{k}=1, \sum_{k=1}^{n} m_{k} \alpha_{k}=\alpha_{0}$, $|z|>1$. If $z$ is a zero of

$$
\frac{P^{\prime}(z)}{P(z)}=A(\neq 0), \quad P(z)=\prod_{k=1}^{n}\left(z-\alpha_{k}\right)^{m_{k}},
$$

then we have

$$
|z-1 / A| \leqq \frac{1+\left|\alpha_{0}\right||z|}{|z|+\left|\alpha_{0}\right|}
$$

If $\alpha_{0}=0$, Theorem 13 is equivalent to Theorem 12. We note that in Theorem 13 we have $\left|\alpha_{0}\right|<1,|\alpha(z)|<1$ except in the single case $\alpha_{k}=\alpha_{0}$ for all $k$, a case that can be treated readily by itself.

Henceforth we give proofs only for the case $\left|\alpha_{0}\right|<1$. To prove Theorem 13, we notice as in [12] that if $|z|>1$ and $z$ satisfies (18), then the function

$$
\beta(z)=\frac{\alpha(z)-\alpha_{0}}{1-\bar{\alpha}_{0} \alpha(z)}
$$

is analytic for $|z|>1$ with (by Lemma 2) $|\alpha(z)|<1,|\beta(z)|<1$ and hence (compare [11]) $\alpha(\infty)=\alpha_{0}, \beta(\infty)=0$. Schwarz's Lemma then asserts $|\beta(z)| \leqq 1 /|z|$ whence by use of various algebraic inequalities related to linear transformations of the complex variable,

$$
|\alpha(z)| \leqq \frac{1+\left|\alpha_{0}\right||z|}{|z|+\left|\alpha_{0}\right|}
$$


Equation (18) can be written

$$
\frac{P^{\prime}(z)}{P(z)}=\sum_{k=1}^{n} \frac{m_{k}}{z-\alpha_{k}}=A, \quad \frac{1}{z-\alpha(z)}=A, \quad z-\frac{1}{A}=\alpha(z)
$$

and (19) follows from (20).

If we modify (18) so that $A=0$, all zeros of the modified equation lie in the closed unit disk, which is essentially the well-known theorem of Lucas relative to the zeros of the derivative of a polynomial.

The locus of $z$ as defined by (19) with the sign " $\leqq$ " replaced by " $=$ " is an algebraic curve of which the part exterior to the unit disk lies in the open disk $|z-1 / A|<1$, since the second member of (19) is less than unity when $|z|>1$. However, we have

COROLlary 8. Suppose in Theorem 13 we have $q>0,|z| \geqq 1+q+q\left|\alpha_{0}\right|$, and equation (18). Then we have

$$
|z-1 / A| \leqq\left(1+q\left|\alpha_{0}\right|\right) /(1+q)
$$

the second member of (21) is clearly less than unity.

For brevity we shall omit the proof. Theorem 12, Theorem 13, and the Corollary 8 all represent an improvement, in some sense, over Theorem 11.

Theorems 11-13 are wholly analogous to a different situation [10].

THEOREM 14. Suppose $\left|\alpha_{k}\right| \leqq 1$ for $k=1,2, \ldots, n$ with $\sum_{k=1}^{n} \alpha_{k}=n \alpha_{0}$. If the constant $C$ is arbitrary we set

$$
p(z)=\prod_{k=1}^{n}\left(z-\alpha_{k}\right)-C .
$$

Then for $|z| \geqq 1$ all zeros of $p(z)$ lie in the $n$ sets of regions (as $C^{1 / n}$ takes all $n$ values)

$$
|z|\left[\left|z-C^{1 / n}\right|-\left|\alpha_{0}\right|\right]+\left|\alpha_{0}\right|\left|z-C^{1 / n}\right| \leqq 1 .
$$

We omit the proof of this theorem which follows along the same lines as the proof of Theorem 13.

\section{REFERENCES}

1. G. M. Goluzin, Geometrische Funktionentheorie, Deutscher Verlag der Wissenschaften, Berlin, 1957.

2. C. A. Kasyaniuk, Estimate of the curvature of level curves by a conformal mapping with bounded distortion, Izv. Vysš. Učebn. Zaved. Matematika 6 (61) (1967), 54-58.

3. Allan K. Krall, On the real parts of zeros of exponential polynomials, Bull. Amer. Math. Soc. 70 (1964), 291-292.

4. Morris Marden, Geometry of polynomials, Math. Surveys, No. 3, Amer. Math. Soc., Providence, R. I., 1966.

5. Zeev Nehari, Conformal mapping, McGraw-Hill, New York, 1952.

6. Z. Rubinstein, Some results in the location of zeros of polynomials, Pacific J. Math. 15 (1965), 1391-1395. 
7. Dorothy Browne Shaffer, Distortion theorems for lemniscates and level loci of Green's functions, J. Analyse Math. 17 (1966), 59-70.

8. G. Szegö, Bemerkungen zu einem Satz von J. H. Grace über die Wurzeln algebraische Gleichungen, Math. Z. 13 (1922), 28-55.

9. J. L. Walsh, On the location of the roots of certain types of polynomials, Trans. Amer. Math. Soc. 24 (1922), 163-180.

10. - A theorem of Grace on the zeros of polynomials revisited, Proc. Amer. Math. Soc. 15 (1964), 354-360.

11. - The location of the zeros of the derivative of a rational function, revisited, J. Math. Pures Appl. 43 (1964), 353-370.

12. J. L. Walsh and Z. Rubinstein, On the location of the zeros of a polynomial whose center of gravity is given, J. Analyse Math. 12 (1964), 129-142.

Clark University,

WORCESTER, MASSACHUSETTS

UNIVERSITY OF MARYLAND,

College Park, Maryland 